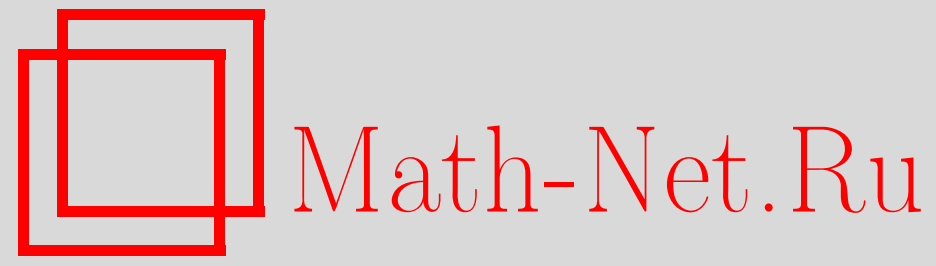

М. С. Бичегкуев, Интегральные операторы, порожденные оператором взвешенного сдвига, $M a-$ тем. заметки, 1996, том 59, выпуск 3, 452-454

DOI: https://doi.org/10.4213/mzm1734

Использование Общероссийского математического портала MathNet.Ru подразумевает, что вы прочитали и согласны с пользовательским соглашением http://www . mathnet.ru/rus/agreement

Параметры загрузки:

IP : 54.210 .77 .194

26 апреля 2023 г., $17: 38: 33$ 


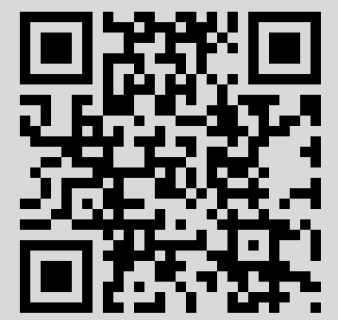




\section{ИНТЕГРАЛЬНЫЕ ОПЕРАТОРЫ, ПОРОЖДЕННЫЕ ОПЕРАТОРОМ ВЗВЕШЕННОГО СДВИГА}

\section{М. С. Бичегкуев}

В работе вводится класс интегральных операторов, ядра которых порождены операторами взвешенного сдвига и изучается их ограниченность в пространствах Лебега.

Введем следующие обозначения: $L_{p}^{+}=L_{p}(0, \infty), L_{p}=L_{p}(-\infty, \infty)$ - лебеговы пространства измеримых функций на полуоси $(0, \infty)$, вещественной прямой $(-\infty, \infty)$, суммируемых со степенью $p \in[1, \infty)$ с нормами $\|\cdot\|_{p}^{+}$и $\|\cdot\| p$ соответственно; $L_{p}^{+}\left(\alpha^{q}\right), q \in(-\infty, \infty), p \in[1, \infty)$ - весовое пространство Лебега с нормой

$$
\|f\|_{p, q}^{+}=\left\|\alpha^{q / p} \cdot f\right\|_{p}^{+} .
$$

Через $p^{\prime}$ обозначим сопряженный показатель для $p \geqslant 1$, т.е. $1 / p+1 / p^{\prime}=1$.

В качестве весовой функции будем рассматривать непрерывную функцию $\alpha=\alpha(t), t \in(0, \infty)$, которая удовлетворяет следующим условиям: $0 \leqslant \alpha(t) \leqslant 1$, $\alpha(t)=1$ при $t \geqslant d\left(0<d-\right.$ фиксированное число); $\lim _{t \rightarrow+0} \alpha(t)=0$; $\lim _{t \rightarrow+0} \int_{t}^{d} \alpha^{-1}(\tau) d \tau=\infty$. По функции $\alpha=\alpha(t)$ построим функцию

$$
x=\varphi(t)=\int_{t}^{d} \alpha^{-1}(\tau) d \tau:(0, \infty) \rightarrow(-\infty, \infty) .
$$

Обозначим через $t=\psi(x)$ функцию, обратную к $x=\varphi(t)$, а через $\gamma=\gamma(t, s)$ функцию, определяемую тождеством

$$
\varphi(\gamma(t, s))=\varphi(t)-\varphi(s) .
$$

Положим

$$
R_{t}^{p} k(s)= \begin{cases}\left(\frac{\alpha(\gamma(t, s))}{\alpha^{p-1}(s)}\right)^{1 / p} k(\gamma(t, s)) & \text { при } 1 \leqslant p<\infty, \\ \frac{k(\gamma(t, s))}{\alpha(s)} & \text { при } \quad p=\infty,\end{cases}
$$

где $\gamma=\gamma(t, s)$ - функция из тождества (1). Оператор (2) представляет собой оператор взвешенного сдвига. В частности, при $d<s<t$ оператор (2) совпадает с оператором "обычного" сдвига. 
Из определения $G_{\alpha, p}[1]$ и $R_{t}^{p}$ непосредственно получаем

$$
G_{\alpha, \infty}^{t \rightarrow x} G_{\alpha, p^{\prime}}^{s \rightarrow y}\left[R_{t}^{p} k(s)\right]=G_{\alpha, p}[k](x-y) .
$$

Свойство оператора (2) характеризует

ЛЕмма. Если $f, g$-измеримье функиии на $(0, \infty)$, то для любого $p \in[1, \infty]$ справедливо равенство

$$
\int_{0}^{\infty} R_{t}^{p} f(s) g(s) d s=\int_{0}^{\infty} f(s) R_{t}^{p} g(s) d s .
$$

Введем оператор

$$
V_{k, p} f(t)=\int_{0}^{\infty} R_{t}^{p} k(s) f(s) d s
$$

где $k(s), s>0,-$ фиксированная функция и $p \in[1, \infty]$. Оператор (4) представляет собой интегральный оператор, ядро которого порождено оператором взвешенного сдвига (2).

Применяя к обеим частям (4) оператор $G_{\alpha, \infty}^{t \rightarrow x}$ и делая замену $s=\psi(y)$ в правой части (4), с учетом (3), получим формулу

$$
G_{\alpha, \infty}^{t \rightarrow x}\left[V_{k, p} f\right](x)=\int_{-\infty}^{\infty} G_{\alpha, p}[k](x-y) G_{\alpha, p}[f](y) d y,
$$

которая устанавливает связь между оператором (4) и интегральньм оператором свертки [2]. Условие ограниченности оператора (4) в $L_{q}^{+}$дает

Tеорема 1. Ecлu $1 \leqslant p \leqslant q<\infty u k \in L_{1}^{+}\left(\alpha^{-1 / p^{\prime}}\right)$, mo onepamop $(4)$ является ограниченным в $L_{q}^{+}$, причем $\left\|V_{k, p} \mid L_{q}^{+} \rightarrow L_{q}^{+}\right\| \leqslant\|k\|_{1,-1 / p^{\prime}}^{+}$.

СледСтвиЕ. Пусть $k \in L_{1}^{+}\left(\alpha^{-1 / p^{\prime}}\right) u 1 \leqslant p \leqslant q<\infty$. Тогда оператор

$$
U_{k, p, q} f(t)=\alpha^{-1 / q}(t) V_{k, p} f(t)
$$

является ограниченным в $L_{p}^{+}$, причем

$$
\left\|U_{k, p, q} \mid L_{p}^{+} \rightarrow L_{p}^{+}\right\| \leqslant\|k\|_{1,-1 / p^{\prime}}^{+}
$$

Используя критерий регулярности интегрального оператора свертки [3] доказывается следующая

ТЕОРема 2. Oператор (5) регулярен в $L_{p}^{+}$тогда и только тогда, когда $k \in L_{1}^{+}\left(\alpha^{-1 / p^{\prime}}\right)$.

Рассмотрим оператор

$$
V_{k, p}^{\#} g(s)=\int_{0}^{\infty} R_{t}^{p} k(s) g(t) d t
$$

который будем назьвать транспонированным по отношению к оператору (4).

Tеорема 3. Пусть $1 \leqslant p<\infty u k \in L_{1}^{+}\left(\alpha^{-1 / p^{\prime}}\right)$. Tогда оnepamop $V_{k, p}^{\#}$ действует в $L_{p^{\prime}}^{+}$и совпадает с сопряжснным $\kappa$ оператору $V_{k, p}$.

ДоКАЗАТЕЛЬСтво проводится методами, развитыми в работах [2], [4]. 


\section{СПИСОК ЦИТИРОВАННОЙ ЛИТЕРАТУРЫ}

1. Глушко В. П., Савченко Ю. Б. // Итоги науки и техн. Матем. анализ. Т. 23. М.: ВИНИТИ, 1985. 2. Коротков В. Б. Интегральные операторы. М.: Наука, 1983. 3. Степанов В. Д. // Сиб. матем. ж. 1974. Т. 15. № 3. С. 693-699. 4. Красносельский М. А., Забрейко П.П., Пустыльник Е. И., Соболевский П. Е. Интегральные операторы в пространствах суммируемых функций. М.: Наука, 1966. 